\title{
Food Prescriptions for Management of Prediabetes and Type 2 Diabetes among Food Insecure Youth: A Pilot Protocol
}

\author{
Jonathan Oswald ${ }^{1}$, Lisa Yazel ${ }^{2}$, Tamara Hannon ${ }^{2}$ \\ ${ }^{1}$ Indiana University School of Medicine; ${ }^{2}$ Indiana University School of Medicine, Department of \\ Pediatrics, Pediatric and Adolescent Comparative Effectiveness Research
}

Introduction: Food access and food insecurity represent significant barriers to healthy eating in low-income communities. Food prescription programs have attempted to address these barriers by providing subsidized access to fresh produce through a physician-community collaboration. However, little is known about the health outcomes associated with food prescriptions, especially among youth with diabetes. To address this, we report data from the past two years of the Veggie Box program, a free produce distribution program of Indy Urban Acres.

Additionally, we propose a feasibility study to 1) establish a food prescription program for food insecure youth with prediabetes and type 2 diabetes through the Veggie Box program and 2) assess health outcomes of youth who receive a prescription for Veggie Box.

Methods: Through 2018 and 2019, the Veggie Box has been distributed weekly to a lowincome community on the eastside of Indianapolis. Participant outcomes have been measured through survey assessment of food and beverage consumption, food insecurity, quality of life indicators, and program satisfaction. The proposed study aims to recruit 70 food insecure families with children aged 10-16 who have prediabetes or type 2 diabetes. Participants will receive a Veggie Box once every two weeks for a total of 12 weeks and will be provided access to a dietitian, health coach, and monthly group diabetes prevention sessions. Participant outcomes will be assessed at baseline and 12 weeks using the same surveys from the past two years with the addition of $\mathrm{BMI}$ and $\mathrm{HbA} 1 \mathrm{c}$ measurements.

Results: The past two years of Veggie Box distribution led to a statistically significant increase in fruit $(p=0.009)$ and vegetable $(p=0.001)$ consumption among Veggie Box recipients. There was not a significant change in reported food security.

Discussion/Conclusion: Early results from the Veggie Box program showed that it increased fruit and vegetable consumption, indicating its potential use as a food prescription for food insecure youth with prediabetes and type 2 diabetes. This study will begin to fill the gap in knowledge related to health outcomes of food insecure youth with diabetes who are provided a food prescription. 